\title{
STRATEGIE-ENTWICKLUNG
}

\section{Erfolgsquelle für soziale Organisationen}

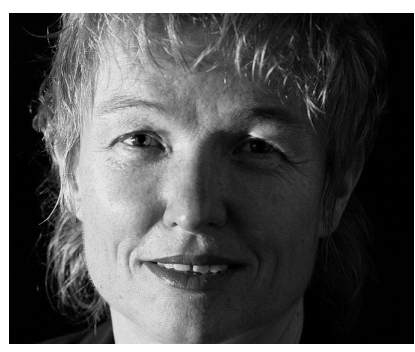

VON ELISABETH BAUER UND GUDRUN SANDER

Elisabeth Bauer ist Betriebswirtin und Sozialarbeiterin. Sie berät Non-Profit-Organisationen und hat Lehraufträge für Sozial-

management an verschiedenen Fachhochschulen in der Schweiz. Internet http://www.elbauer.ch

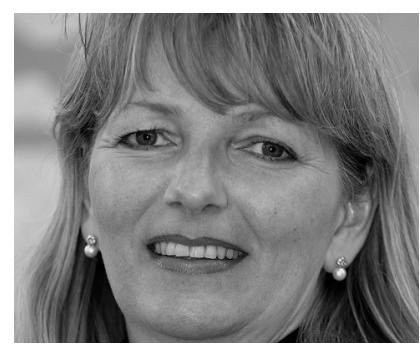

Dr. Gudrun Sander ist Lehrbeauftragte für Betriebswirtschaftslehre und für Gender- und DiversityManagement an der Universität St. Gallen, Lehrbeauftragte für Sozialmanagement an der Zürcher Hochschule für Angewandte Wissenschaften und Lehrbeauftragte für Diversity an der Universität Zürich.

Internet

http://www.es.unisg.ch/wbb
Soziale Organisationen, die sich auf die Megatrends von Gegenwart und Zukunft einstellen wollen, brauchen eine dafür geeignete flexible Unternehmensstrategie. In der Schweiz wurden Führungskräfte von Non-Profit-Organisationen befragt, die einen Kurs für Strategisches Management absolviert haben, welche Erfahrungen sie damit in der Praxis gemacht haben.

Strategisches Management ist hoch im Kurs. Seit 50 Jahren gilt die Strategie als Erfolgsquelle für Unternehmen schlechthin. Auch im Non-Profit-Management hat das strategische Denken inzwischen Einzug gehalten, was sich einerseits in den Vorgaben von Finanzierern zeigt, welche immer häufiger von sozialen Organisationen verlangen, Strategie-Entwicklungsprozesse durchzuführen. Andererseits widerspiegelt sich der Aufschwung des strategischen Managements im Non-Profit-Sektor in neuen Publikationen (1) sowie in neuen Angeboten von Weiterbildungsorganisationen (2). Wozu aber sollen Strategie-Entwicklungsprozesse in sozialen Organisationen gut sein?

\section{Warum Strategie-Entwicklung nötig ist}

Pfeffer und Sutton, zwei renommierte Professoren für Strategisches Management an der kalifornischen Standford University, hinterfragen in ihrem neuesten Werk (3) den anhaltenden Strategie-Boom kritisch. $\mathrm{Zu}$ Recht weisen sie darauf hin, dass Strategie-Spezialisten im Bestreben nach Sicherheit in einer sich ständig wandelnden Umgebung Gefahr laufen, viel zu viel Zeit und Geld in aufwändige Analysen zu investieren, welche schließlich der Organisation herzlich wenig Nutzen bringen, weil die Daten, kaum publiziert, schon wieder veraltet sind.

Von oben diktierte Strategien lassen sich außerdem häufig nicht umsetzen, insbesondere dann nicht, wenn sich die Mitarbeitenden mit der neuen Strategie nicht identifizieren können. Schließlich kann eine einmal beschlossene Strategie eine Organisation dazu verleiten, sich in falscher Sicherheit zu wiegen und wichtige Signale in der Umwelt, welche die gewählte Strategie infrage stellen, zu missachten.

Allerdings gibt es auch sehr bedeutende Argumente dafür, dass Strategie-Entwicklungsprozesse entscheidende Vorteile bringen. Ob im sozialen, kulturellen oder politischen Bereich tätig, jede Organisation muss sich nämlich für eine klare Entwicklungsrichtung entscheiden. Pfeffer und Sutton betonen denn auch, dass es wichtiger ist, »das Richtige zu tun, selbst wenn es nicht perfekt getan wird, als das Verkehrte ausgesprochen gründlich zu tun« (S. 189).

Für jede Organisation ist es deshalb überlebensnotwendig zu begreifen, was sie für ihren Erfolg tun muss, mit anderen Worten, welche Strategie zu implementieren ist, damit sie überleben kann.

\section{Wie Strategie-Entwicklung gelernt werden kann}

Strategische Entscheidungen erfordern von den Führungskräften und der ganzen Organisation spezifisches Knowhow. Da diese Entscheidungen je länger je mehr in einem sich rasch wandelnden, unsicheren und mehrdeutigen Umfeld getroffen werden müssen, kommt dem strategischen Know-how eine wachsende Bedeutung zu.

Wie aber kann sich eine Organisation dieses Know-how erwerben? Dass gerade die Planung und Umsetzung eines StrategieEntwicklungsprozesses diese Fähigkeiten in entscheidender Weise fördert, belegt eine empirische Studie, welche wir im Frühling 2008 im Auftrag des Departementes Soziale Arbeit der Zürcher Hochschule für angewandte Wissenschaften (ZHAW) durchführten.

Mit Hilfe einer elektronischen Lernplattform stellten wir den Absolventinnen und Absolventen aller Kursangebote der Zürcher Hochschule in Strategischem Management zwischen 2003 und 2007 einen elektronischen Fragebogen zu. Damit konnten 150 Führungskräfte von sozialen Organisationen erreicht werden. Nach einmaligem Nachfassen füllten 51 Personen den Fragebogen aus (Rücklaufquote: $34 \%)$. 
In fast drei Viertel der antwortenden Organisationen wurde nach dem Kurs ein Strategie-Entwicklungsprozess durchgeführt (25 Personen) oder ist einer geplant (10 Personen). Wie wurde in jenen Organisationen vorgegangen, welche einen Strategie-Entwicklungsprozess durchgeführt haben? Der Prozess erwies sich mehrheitlich als aufwändig und dauerte ein Jahr oder länger. Die meisten Organisationen wählten ein eher analytisches und weniger intuitives Vorgehen und führte den Prozess selbständig $(50 \%)$ oder punktuell mit externer Fachbegleitung (46\%) durch.

\section{Was Strategie- \\ Entwicklungsprozesse in sozialen Organisationen bewirken}

Die Resultate der Strategie-Entwicklungsprozesse, von denen die Befragten berichten, sind beeindruckend. Mehrheitlich kam es zu

- einer Stärkung der Identität der Organisation

- klaren, transparenten und breit akzeptierten Zielen

- einer Verbesserung der Strukturen und Prozesse

- einer Verbesserung der Zusammenarbeit mit der Trägerschaft

- einer verstärkten Kundenorientierung

Auf der negativen Seite schlagen der hohe Zeitaufwand und Führungsmängel wie fehlender Einbezug der Mitarbeitenden zu Buche.
Die positiven Wirkungen des StrategieEntwicklungsprozesses werden vom Selbstbild der antwortenden Organisationen untermauert. Diejenigen Führungskräfte, welche einen Strategie-Entwicklungsprozess durchgeführt haben, bewerten nämlich die aktuellen Ressourcen ihrer Organisation durchwegs besser als jene 26 Personen, welche keinen Prozess durchführten. Besonders die Fähigkeiten, eine gemeinsame Vision zu haben, die Prozesse kontinuier- (z. B. Erreichung der festgelegten Ziele, Qualität der erbrachten Leistungen) aus, während die übrigen Personen am meisten prozessbezogene Faktoren bestimmten (z. B. gemeinsam festgelegte Ziele haben, eine gemeinsame Vision haben). Somit orientieren sich Führungskräfte mit StrategieErfahrung eher an wirkungsorientierten Zielen, während die übrigen Personen ihr Handeln eher an prozessorientierten Zielen ausrichten.

\section{"Ein Strategie-Entwicklungsprozess schärft den Blick aller Mitarbeitenden auf Stärken und Schwächen der Organisation «}

lich zu verbessern, flexibel und innovationsfähig zu sein oder kundenorientiert zu arbeiten scheinen Organisationen mit Strategie-Erfahrung markant besser zu beherrschen (vgl. Abb. 1).

Neben optimierten Ressourcen dürften die Strategie-Entwicklungsprozesse in den befragten Organisationen auch zu Veränderungen in der Handlungsorientierung geführt haben. Auf die Aufforderung, aus 24 verschiedenen Fähigkeiten die fünf zukünftig Wichtigsten zu nennen, wählten nämlich Führungskräfte mit Strategie-Erfahrung am häufigsten wirkungsbezogene Faktoren

\section{Welche Lernprozesse Strategie- Entwicklung auslöst}

Ein weiterer deutlicher Unterschied betrifft die Wahrnehmung von Entwicklungen in der Umwelt. Die Untersuchungsresultate legen nahe, dass Führungskräfte mit Strategie-Erfahrung ihr Umfeld bedeutend differenzierter wahrnehmen als die übrigen befragten Personen. Daraus lässt sich schließen, dass die Durchführung eines Strategie-Entwicklungsprozesses die Fähigkeiten verbessert, sich mit zukünftigen Ent-

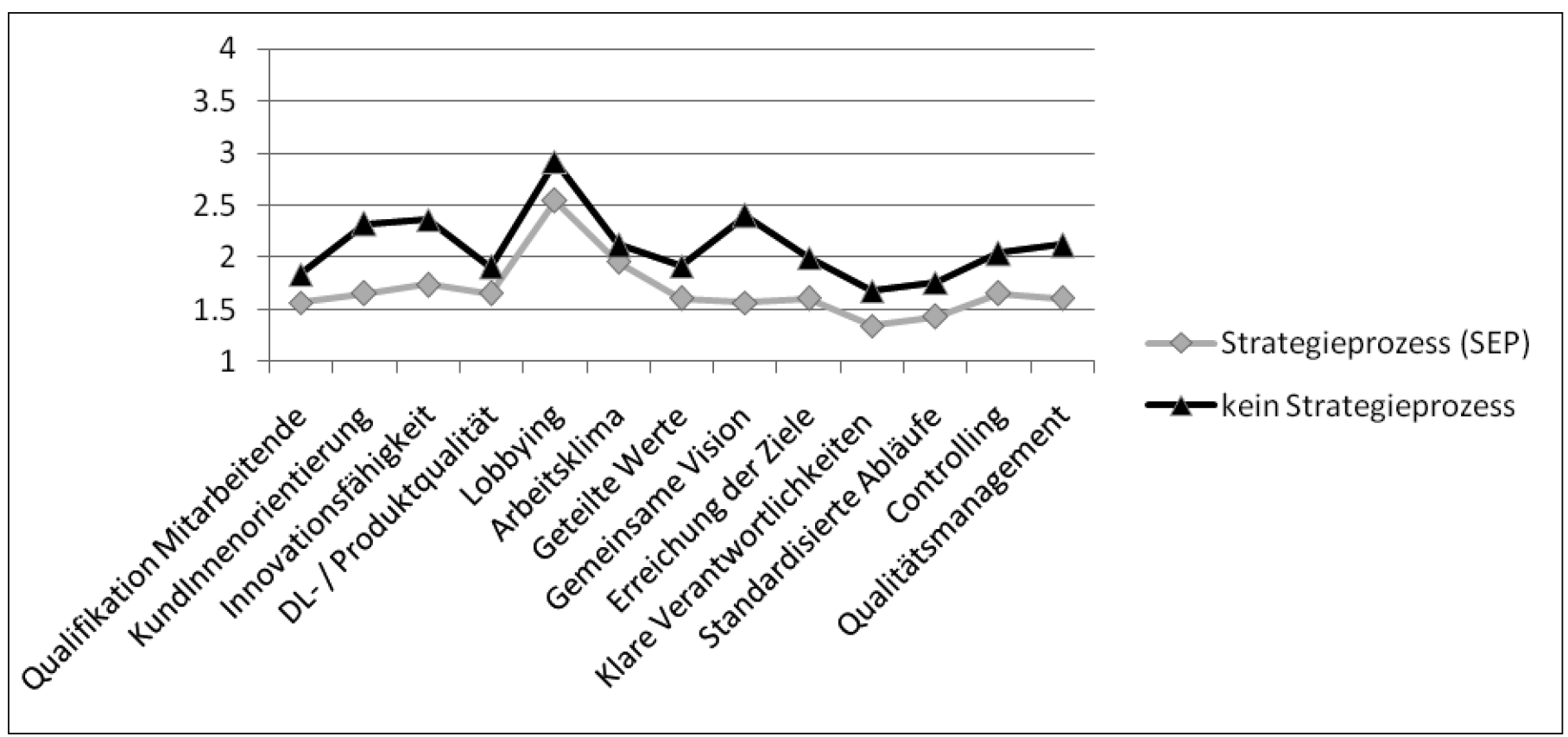

Abb. 1: Führungskräfte, die einen Strategie-Entwicklungsprozess durchgeführt haben, bewerten die Ressourcen ihrer Organisation durchwegs besser als jene, welche keinen Prozess durchführten.

Legende: $n(S E P)=$ 23, $n$ (kein SEP) = 25; Mittelwerte. 1 = sehr gut/vorhanden; 2 = gut/teilweise vorhanden; 3 = mittelmäßig/kaum vorhanden; 4 = schlecht/nicht vorhanden. 
wicklungen und Anforderungen kritisch auseinanderzusetzen.

Das Lernen durch Strategie-Entwicklung beschränkt sich jedoch nicht auf die Führungskräfte, sondern betrifft die ganze Organisation. So berichten die Befragten, dass sie ihre Leitungskompetenzen sowie ihre eigene strategische Denkart verbessert hätten. Darüber hinaus habe aber auch die gesamte Organisation ihren Blick auf die Entwicklungen im Umfeld sowie auf die eigenen Stärken und Schwächen schärfen können. Besonders in jenen Organisationen, welche die Mitarbeitenden in den Prozess einbezogen, ist es zu einer Bündelung der Kräfte und zu einer neuen Art der Kommunikation gekommen. Dadurch sind Mitverantwortung und Engagement gewachsen.

Mit diesen Aussagen beschreiben die befragten Führungskräfte nicht nur Wirkungen des "Lernens 1. Ordnung " (4) (z. B. lernen, ein Instrument einzusetzen), sondern ebenfalls des »Lernens 2. Ordnung « (d. h. lernen zu lernen). Die mit Lernprozessen 2. Ordnung erworbenen Metafähigkeiten wie beispielsweise ein geschärfter Blick auf die Entwicklungen im Umfeld haben im letzten Jahrzehnt auch für Non-Profit-Organisationen enorm an Bedeutung gewonnen, weil sich das Umfeld immer rascher wandelt. Für die Bewältigung der wachsenden Unsicherheiten und Mehrdeutigkeiten sind Fähigkeiten 2. Ordnung unabdingbar. Die möglichen Zusammenhänge zwischen den verschiedenen individuellen und kollektiven Lernprozessen können grafisch dargestellt werden (vgl. Abb. 2).

\section{Wie sich Strategie- \\ Entwicklungsprozesse durchführen lassen}

Die Resultate unserer empirischen Untersuchung legen nahe, dass es sich auch für soziale Organisationen auszahlt, StrategieEntwicklungsprozesse zu planen und umzusetzen; ja, dass es für sie in Zukunft gar überlebensnotwendig sein wird, solche Prozesse durchzuführen. Außerdem macht die Analyse deutlich, dass Strategie-Entwicklung explizit als Lernprozess der gesamten Organisation zu konzipieren ist und die Mitarbeitenden gezielt einzubeziehen sind. Konkret bedeutet dies, ein klares, strukturiertes und systematisches Vorgehen zu wählen und gleichzeitig Offenheit zu zeigen für Initiativen von der Basis und für das $\mathrm{Zu}-$ fällige.

Von besonderem Gewicht ist deshalb die Initiierungsphase, also die Planung des gesamten Prozesses. Indem die Führungskräfte in einem ersten Schritt die Verantwortlichkeiten klären, den Beteiligungsgrad der Mitarbeitenden bestimmen, das Timing bezeichnen, die notwendigen personellen und

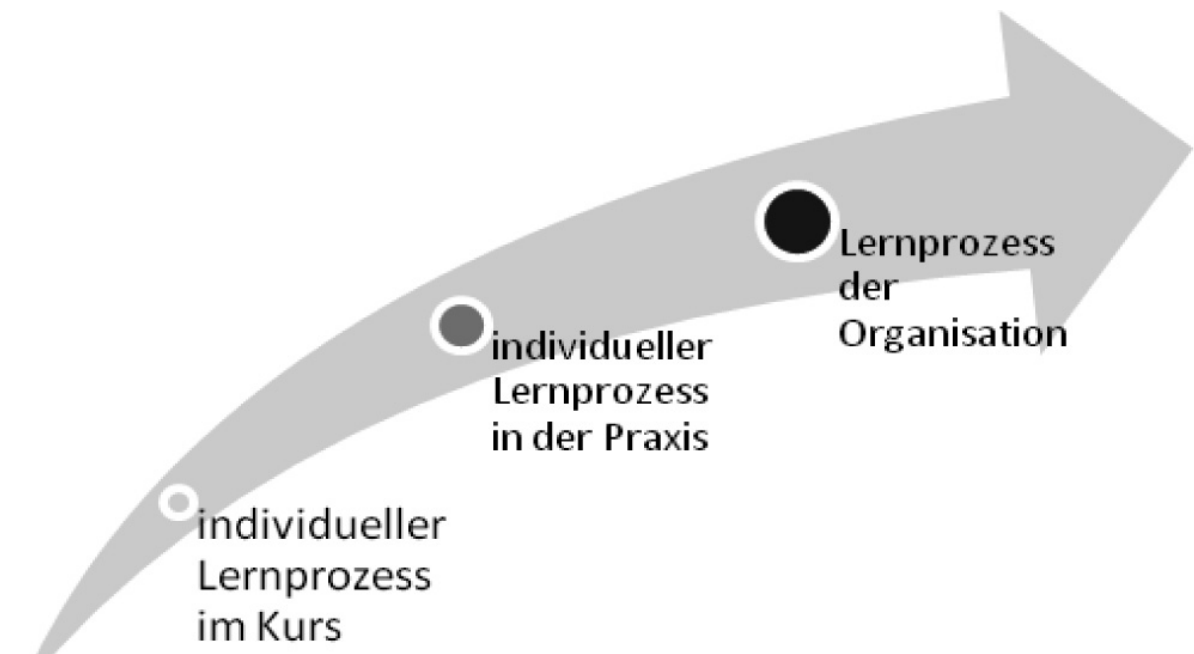

Abb. 2: Zwischen individuellen und kollektiven Lernprozessen gibt es sich gegenseitig bedingende Zusammenhänge.

finanziellen Ressourcen festlegen, Instrumente und Methoden auswählen und die geplanten Schritte so weit wie möglich konkretisieren, verfertigen sie sich einen gemeinsamen, flexiblen Rahmen für den kollektiven Lernprozess.

Dieser Rahmen leitet sie durch die anschließende Analysephase, in welcher sie einerseits die Chancen und Risiken im Umfeld und andererseits die Stärken und Schwächen der eigenen Organisation aufdecken. Wie können die Chancen durch den Einsatz der Stärken genutzt werden? Wie lassen sich Bedrohungen durch interne Stärken mildern? Dabei ist es wichtig, die konventionellen Denkbahnen immer wieder zu verlassen und auch vermeintliche Seitenwege auszuprobieren. In der Konzeptionsphase werden dann konkrete Strategien abgeleitet, welche es - last but not least - in der Umsetzungsphase umzusetzen gilt.

\section{Fazit}

Eine gute Weiterbildung bringt den Führungskräften das nötige Rüstzeug, um selber einen Strategie-Entwicklungsprozess planen und durchführen zu können.

Wenn die Führungskräfte ihren individuellen Lernprozess in der Weiterbildung am konkreten Umsetzungsfall in ihrer eigenen Organisation erproben, lösen sie damit Lernprozesse der gesamten Organisation aus. Nicht nur die Erarbeitung und Umsetzung einer Strategie sind als » Lernergebnis « zu erwarten, sondern auch verbesserte Metafähigkeiten der Organisation, wie etwa den Blick für die Entwicklungen im Umfeld zu schärfen, die Selbstwirksamkeit der Organisation differenzierter wahrzunehmen und eine wirkungsbezogene Zielerreichung zu fördern.
Strategie-Entwicklungsprozesse sind also weit mehr als das Produzieren von Hochglanzbroschüren für die Schubladen.

\section{Anmerkungen}

(1) U. a. Sander, G./Bauer, E. (2006). Strategieentwicklung kurz und klar. Das Handbuch für Non-Profit-Organisationen. Bern: Haupt. Bätscher, R./Ermatinger, J. (2004). Strategieentwicklung in Sozialinstitutionen. Ein Leitfaden für die Praxis. Zürich: Versus. Sieper, M. (2008). Strategische Planung in Nonprofit-Organisationen. Hamburg: Diplomica.

(2) Z. B. Strategieentwicklung kurz und klar. Der Kurs zum Buch. Zürcher Hochschule der angewandten Wissenschaften (ZHAW), Departement Soziale Arbeit.

(3) Pfeffer, J./Sutton, R.I. (2007). Harte Fakten, gefährliche Halbwahrheiten und absoluter Unsinn. München: Pearson Business.

(4) Vgl. Schwaninger, M. (2006). Intelligent Organizations. Powerful Models for Systemic Management. Berlin: Springer. 